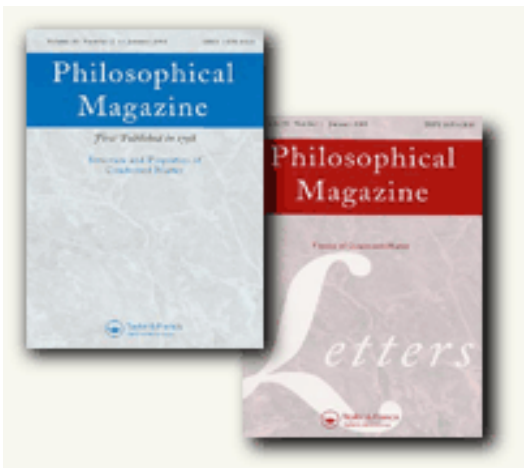

\title{
Coulomb repulsive correlation in systems with radial confinement: \\ quantum dots and the Overhauser model in an external magnetic field
}

\begin{tabular}{|c|c|}
\hline Journal: & Philosophical Magazine \& Philosophical Magazine Letters \\
\hline Manuscript ID: & TPHM-04-Dec-0143.R1 \\
\hline Journal Selection: & Philosophical Magazine \\
\hline $\begin{array}{l}\text { Date Submitted by the } \\
\text { Author: }\end{array}$ & 06-May-2005 \\
\hline Complete List of Authors: & $\begin{array}{l}\text { Avetisyan, Artak; University of Cyprus, Department of Physics } \\
\text { Moulopoulos, Konstantinos; University of Cyprus, Department of } \\
\text { Physics } \\
\text { Djotyan, Anahit; Yerevan State University, Department of Physics }\end{array}$ \\
\hline Keywords: & $\begin{array}{l}\text { low-dimensional structures, electron energy states, correlated } \\
\text { systems }\end{array}$ \\
\hline Keywords (user supplied): & quantum dots, acceptor states, GaAs \\
\hline
\end{tabular}

\section{今scholaroNE \\ Manuscript Central}




\begin{abstract}
The ground state correlation energy of an electron-negative ion system in a spherical quantum dot (QD) with parabolic confinement in an external homogeneous magnetic field is investigated. Both cases of finite and infinite potential barrier on the QD surface are considered. The theoretical analysis is carried out using a variational approach. The ground state energy of a twoelectron system in a QD with harmonic-oscillator potential in a magnetic field is also determined as a function of the strength of the magnetic field and parabolic confinement; the influence of the finite potential barrier on the correlation energy of the system is also discussed. A first investigation of electron-electron correlation in a two-dimensional (2D) system with the screened Coulomb potential, proposed by Overhauser (1995), but in the presence of a perpendicular magnetic field is shown to be another important application of the proposed variational wave functions.
\end{abstract}

Keywords: quantum dots, parabolic potential, acceptor states, correlation energy, GaAs PACS numbers: 73.21.La, 73.43.Nq, 71.70.Di

\title{
§1. Introduction
}

Over the last decade, materials development based on atomic layer growth, doping and gating techniques have led to semiconductor QD systems in which a single electron can be injected into a QD, and a single QD can be probed optically (see [1,2] and references therein). Moreover, some QD layout enables to study any fixed number of electrons from zero to over fifty [3]. Obviously, QDs can contain impurity centers (donors or acceptors). The presence of the impurity states in nanostructures dramatically influences both the electronic mobility and their electronic and optical properties $[4,5]$. In such systems it is of great importance to understand how the electron-electron (or electron-negative ion) interaction controls the physics. It is well known that the Coulomb interaction is enhanced by the electron confinement and the strongest effect is expected for the QDs.

The problem of two electrons in a spherically symmetric QD with the harmonic-oscillator potential was studied in papers [6-7] and analytical solutions were found for a particular set of oscillation frequencies. The exact initial two-electron states of the parabolic potential well have been used in [8] to calculate the double photoemission spectra of a QD with the typical parameters. The interplay between a confining potential, approximated by the harmonic oscillator $[9,10]$ and the repulsive Coulomb interaction leads to interesting physical effects in a magnetic field even in the case of twoelectron QDs [11, 12], and more generally in the case of a few and multielectron QDs [13-15].

Several studies of the two-electron system behaviour in parabolic QDs in a magnetic field were performed in the last decade [16-23]. In the majority of these papers the problem of two electrons (or an electron-negative ion system) in semiconductor QDs in a magnetic field has been investigated in 2D harmonic-oscillator potential by numerical simulations or by series expansion methods.

However for this potential there exist only bound states and this leads to the fundamental physical failure of this model, namely, the binding and dissociation processes cannot be described with its use. For the real QD the confinement potential has finite depth and a QD can be charged by the finite numbers of electrons which determine a quantum "capacity" of QD [24]. The two-electron problem inside a flat circular hard wall potential of finite height was studied in [25].

\footnotetext{
*E-mail:avetis@ucy.ac.cy, artakav@ysu.am
} 
The principal aim of this work is to develop a variational approach for obtaining the ground state energy of an electron-negative ion (or a two-electron) system in a spherical QD under parabolic potential in a magnetic field, as well as to examine the sensitivity of the energy of the system to the different confinement conditions on the QD surface.

Similar trial wave functions are also used in the present work for investigation of the electronelectron correlation in a 2D system with the screened Coulomb potential proposed by Overhauser [26] but in an external magnetic field. The Overhauser model has been successfully used for determining the pair distribution function for interacting electron gas in a rigid uniform neutralizing background [26, 27]. Now, in the additional presence of an external homogenous magnetic field the two-electron system will have confined states, and it is a natural continuation to use the developed variational method for this problem as well.

The knowledge of the analytic form of two correlated electron wave function in a magnetic field can also be useful in constructing a trial many-body wave functions for the investigation of possible novel phases such as paired electron crystal [28, 29] and Wigner molecule phases in QDs [30].

Section 2 contains an outline of the underlying theory and gives closed analytical expressions that determine the QD ground state energy for various cases. Section 3 presents our results together with a discussion of relevant physical points. Section 4 presents our main conclusions. Two exactly soluble models that serve as useful limiting cases for our results are given in two Appendices.

\section{§2. Theory}

\subsection{QD with an infinite potential barrier}

Semiconductor QDs can confine a few electrons in three dimensions [3, 11]. By injecting of two electrons in a QD containing an acceptor impurity one can reach the electron-negative ion system in the QD (the hole generated by acceptor as a free charge carrier can be removed from the QD, or recombined with injected electron in the QD). The Hamiltonian of a system consisting of an electron in the field of a negative ion (acceptor impurity) fixed at the centre of a spherical QD with the radius $d$ (in units of the length $a_{B}^{*}=\hbar^{2} \kappa / m^{*} e^{2}$, the energy $R^{*}=e^{2} / 2 \kappa a_{B}^{*}$ ) is:

$$
H=-\nabla^{2}+\frac{2}{r}+V(r)
$$

where $V(r)=\gamma_{p}^{2} r^{2}$, when $r \leq d$ and $V(r)=\infty$ for $r>d ; \gamma_{p}=\hbar \omega_{p} / 2 R^{*}$ is the dimensionless measure of QD parabolic potential with the harmonic oscillator frequency $\omega_{p}, m^{*}$ is the effective mass of electron, $\kappa$ is the dielectric constant of semiconductor (GaAs) material inside the QD.

Based on an exact solution of the system with Coulomb repulsive interaction in 3D harmonic potential [6], we have chosen the ground state variational wave function $\psi$ of the system in a form:

$$
\psi=N \exp \left(-\lambda r^{2}\right)(1+\delta r) \frac{J_{1 / 2}\left(\alpha_{1} r\right)}{\sqrt{r}},
$$

that satisfies the boundary condition $\psi(r=d)=0 ; \lambda, \delta$ are the variational parameters and $\alpha_{1}=k_{1} / d, k_{1}$ is the first root of the Bessel function $J_{1 / 2}\left(\alpha_{1} r\right)$. The normalization constant $N$ is:

$$
N^{-2}=4 \pi A, \quad A=\int_{0}^{d} e^{-2 \lambda r^{2}}(1+\delta r)^{2} J_{1 / 2}^{2}\left(\alpha_{1} r\right) r d r
$$

For the ground state energy of the system after some algebraic manipulations we find:

$$
E=\alpha_{1}^{2}+6 \lambda+A^{-1}\left(I_{1}+I_{2}\right)
$$




$$
I_{2}=\int_{0}^{d}\left\{\left(2 \alpha_{1} \delta r(1+\delta r)-4 \alpha_{1} \lambda r^{2}(1+\delta r)^{2}\right) J_{1 / 2}\left(\alpha_{1} r\right) J_{3 / 2}\left(\alpha_{1} r\right)\right\} \exp \left[-2 \lambda r^{2}\right] d r .
$$

\subsection{QD with a finite potential barrier}

In this case, the Hamiltonian of the system has the form of equation (1) with the potential

$$
V(r)=\gamma_{p}^{2} r^{2} \text {, when } r \leq d \text {, and } V(r)=U_{0}\left(U_{0} \geq \gamma_{p}^{2} d^{2}\right) \text { for } r>d .
$$

The wave function $\psi$ and its radial derivative should be continuous at the QD boundary. We have chosen $\psi$ in a form that satisfies this requirement (see, for example, [31]), namely

$$
\psi=N \frac{\exp \left(-\lambda r^{2}\right)(1+\delta r)}{r^{1 / 2}}\left\{\begin{array}{l}
J_{1 / 2}\left(\alpha_{1} r\right), \quad r<d \\
c_{k} K_{1 / 2}\left(\beta_{1} r\right), \quad r \geq d
\end{array},\right.
$$

where $c_{k}=\left\{J_{1 / 2}\left(\alpha_{1} d\right) / K_{1 / 2}\left(\beta_{1} d\right)\right\}, K_{1 / 2}\left(\beta_{1} r\right)$ is the modified Bessel function of the second kind, $\alpha_{1}^{2}=\varepsilon_{1}, \beta_{1}^{2}=U_{0}-\alpha_{1}^{2}$ and $\varepsilon_{1}$ is the lowest energy of a free electron in a QD with the potential barrier $U_{0}$ (in the absence of Coulomb interaction and parabolic potential $\gamma_{p}^{2} r^{2}$ ) obtained from [32]:

$$
-\sqrt{U_{0}-\varepsilon} \tan d \sqrt{\varepsilon}=\sqrt{\varepsilon} .
$$

For the ground state energy of the system we obtain the expression (9) with new $A^{-1}=4 \pi N^{2}$ :

$$
E=\alpha_{1}^{2}+6 \lambda+A^{-1}\left\{I_{1}+I_{2}+\left[J_{1 / 2}\left(\alpha_{1} d\right) / K_{1 / 2}\left(\beta_{1} d\right)\right]^{2}\left(L_{1}+L_{2}\right)\right\},
$$

where $I_{1}$ and $I_{2}$ are given by expression (5) with new values of $\alpha_{1}$ obtained from equation (8) and

$$
\begin{gathered}
L_{1}=\int_{d}^{\infty}\left\{\left(2-4 \lambda^{2} r^{3}\right)(1+\delta r)^{2}+\left(4 \lambda \delta r^{2}-2 \lambda\right)(1+\delta r)\right\} K_{1 / 2}^{2}\left(\beta_{1} r\right) \exp \left[-2 \lambda r^{2}\right] d r, \\
L_{2}=\int_{d}^{\infty}\left\{\left(2 \beta_{1} \delta r(1+\delta r)-4 \beta_{1} \lambda r^{2}(1+\delta r)^{2}\right) K_{1 / 2}\left(\beta_{1} r\right) K_{3 / 2}\left(\beta_{1} r\right)\right\} \exp \left[-2 \lambda r^{2}\right] d r .
\end{gathered}
$$

\subsection{QD in the presence of external magnetic field with a finite potential barrier}

The Hamiltonian of the system consisting of an electron in the field of a negative ion at the centre of a spherical QD under a parabolic confining potential $V(r)$ (see equation (6)) and in the presence of a homogeneous magnetic field $\mathbf{B}$ applied along the $z$-direction (we choose $\mathbf{A}(\mathbf{r})=(\mathbf{B} \times \mathbf{r}) / 2$ ) reads

$$
H=-\nabla^{2}+\gamma L_{z}+\frac{1}{4} \gamma^{2} r^{2} \sin ^{2} \theta+\frac{2}{r}+V(r),
$$

where $L_{z}$ is the $z$ - component of the angular momentum operator (in units of $\hbar$ ), and $\gamma$ is a dimensionless measure of $\mathbf{B}, \gamma=\hbar \omega_{B} / 2 R^{*}$, with the cyclotron frequency $\omega_{B}=e B / m^{*} c$. Now, we have chosen the trial wave function with variational parameters $\lambda, \delta$ in the form

$$
\psi_{1}(r, \theta)=\psi(r) \exp \left(-\gamma r^{2} \sin ^{2} \theta / 4\right),
$$

where $\psi(r)$ is given by equation (7), and $g(r, \theta)=\exp \left(-\gamma r^{2} \sin ^{2} \theta / 4\right)$ describes the influence of the 
magnetic field [33]. For the ground state energy of the system we have obtained the analytic expression which is not presented here because of its complicated form.

\subsection{Electron-electron correlation in parabolic QD in the presence of a magnetic field}

The Hamiltonian of a two-electron system in a QD under an infinite range harmonic potential and in a homogeneous magnetic field $\mathbf{B}$ applied along the $z$-direction $\left(\mathbf{A}\left(\mathbf{r}_{i}\right)=\left(\mathbf{B} \times \mathbf{r}_{i}\right) / 2\right)$ can be written as

$$
H=\sum_{i=1,2}\left[\frac{1}{2 m^{*}}\left(\hat{\mathbf{p}}_{i}+\frac{e}{c} \mathbf{A}\left(\vec{r}_{i}\right)\right)^{2}+V\left(r_{i}\right)\right]+\frac{e^{2}}{\kappa\left|\vec{r}_{1}-\vec{r}_{2}\right|} .
$$

Now we introduce relative and centre-of-mass coordinates with new momentum operators:

$$
\vec{r}=\vec{r}_{1}-\vec{r}_{2}, \vec{R}=\frac{1}{2}\left(\vec{r}_{1}+\vec{r}_{2}\right), \hat{\mathbf{p}}=\frac{1}{2}\left(\hat{\mathbf{p}}_{1}-\hat{\mathbf{p}}_{2}\right), \hat{\mathbf{P}}=\hat{\mathbf{p}}_{1}+\hat{\mathbf{p}}_{2} .
$$

Since $\mathbf{B}$ is homogenous, $\mathbf{A}$ is a linear function [19] and we have

$$
\mathbf{A}(\vec{r})=\mathbf{A}\left(\vec{r}_{1}\right)-\mathbf{A}\left(\vec{r}_{2}\right), \mathbf{A}(\vec{R})=\frac{1}{2}\left(\mathbf{A}\left(\vec{r}_{1}\right)+\mathbf{A}\left(\vec{r}_{2}\right)\right) .
$$

In spherical coordinates, using equations (14)-(16) the Hamiltonian for the relative motion is (in units $a_{B r}=\hbar^{2} \kappa / m_{r} e^{2}, R_{B r}=e^{2} / 2 \kappa a_{B r}$ with reduced mass $\left.m_{r}=m^{*} / 2\right)$ :

$$
H=-\nabla^{2}+\gamma L_{z r}+\frac{1}{4} \gamma^{2} r^{2} \sin ^{2} \theta+\gamma_{p}^{2} r^{2}+\frac{2}{r} .
$$

We have chosen the trial wave function with variational parameters $\lambda, \delta$ in the form

$$
\psi=N \exp \left(-\gamma r^{2} \sin ^{2} \theta / 4\right) \exp \left(-\lambda r^{2}\right)(1+\delta r),
$$

For the ground state energy of the system we obtain

$$
\begin{gathered}
\left.E=6 \lambda+\gamma+A^{-1}\left\{J_{1}+J_{2}\right)\right\} \\
J_{1}=\int_{0}^{\infty} \int_{0}^{\pi}\left(\left(\gamma_{p}^{2}-4 \lambda^{2}-2 \lambda \gamma \sin ^{2} \theta\right) r^{3}+2\right)(1+\delta r)^{2} \exp \left[-2 \lambda r^{2}\right] \exp \left(-\gamma r^{2} \sin ^{2} \theta / 2\right) \sin \theta d \theta r d r, \\
J_{2}=\int_{0}^{\infty} \int_{0}^{\pi} 2 \delta\left[\left(2 \lambda+\gamma \sin ^{2} \theta / 2\right) r^{2}-1\right](1+\delta r) \exp \left[-2 \lambda r^{2}\right] \exp \left(-\gamma r^{2} \sin ^{2} \theta / 2\right) \sin \theta d \theta r d r .
\end{gathered}
$$

\subsection{Correlations in $2 \mathrm{D}$ electron gas: Overhauser model in a magnetic field}

In the interacting electron gas problem, a rigid uniform positively charged background maintains electrical neutrality. The screened Coulomb potential in Overhauser model (equation (21) is defined through the use of a neutral spherical cell (in 3D) in relative coordinates [26] with an electron at the cell center. The background inside the cell contributes the parabolic potential (see the second term in equation (21)) due to the background density, which is equal to the electronic number density $n_{0}$. The constant term in equation (21) is such that the total potential on the spherical boundary is made to vanish. For 3D the correct choice of cell radius $a_{O v}$ is: $a_{O v}=r_{s} a_{B}$, where $r_{s}$ is the radius (in units $a_{B}=\hbar^{2} / m e^{2}, m$ is the mass of free electron) of a sphere containing one electron on the average, i.e., $n_{0}=\left(4 \pi r_{s}^{3} a_{B}^{3} / 3\right)^{-1}$. In atomic units, $\left(a_{B}\right.$ and $\left.R_{B}=e^{2} / 2 a_{B}\right)$, the Overhauser potential becomes :

$$
V(r)=\frac{2}{a_{O v}}\left(\frac{a_{O v}}{r}+\frac{1}{2} \frac{r^{2}}{a_{O v}^{2}}-\frac{3}{2}\right), \quad r \leq a_{O v} ; \quad V(r)=0, r>a_{O v} \quad\left(a_{O v}=r_{s}\right) .
$$


The Overhauser approach has also been applied to the 2D electron gas problem $[34,35]$ with the use of a circular cell (disc), with $r_{s}$ being defined now by $n_{0}=\left(\pi r_{s}^{2} a_{B}^{2}\right)^{-1}$. In [34] the exact background contribution was taken into account, which in $2 \mathrm{D}$ is given by a hypergeometric function (see equation (22) below). In [35] an expansion of this function, up to $4^{\text {th }}$ order in $r$ has been used. In 2D there is a complication (because Gauss' law is valid only in 3D): the total potential cannot be made to vanish outside the disc, if the radius of disc is chosen equal to $r_{s} a_{B}$. This difficulty has been physically resolved through a procedure proposed by Nagy [36] in a variational treatment of the total electrostatic energy. The radius $a_{N}$ of the cell was treated as a variational parameter, with its optimum value $a_{N}=\pi^{1 / 2} r_{s} a_{B} / 2$ [36]. Within this model the effective potential is:

$$
V(r)=\frac{2}{r}-\frac{4 a_{N}}{r_{s}^{2}}{ }_{1} F_{2}\left(\frac{1}{2},-\frac{1}{2} ; 1 ; \frac{r^{2}}{a_{N}^{2}}\right), \quad r \leq a_{N} ; \quad V(r)=0, \quad r>a_{N}\left(a_{N}=\pi^{1 / 2} r_{s} / 2\right) .
$$

Now, by separating two particles out of the many-body system, an effective two-electron problem can be defined in 2D plane in an external perpendicular magnetic field, with a Hamiltonian

$$
H=\sum_{i=1,2} \frac{1}{2 m}\left(\hat{\mathbf{p}}_{i}+\frac{e}{c} \mathbf{A}\left(\vec{r}_{i}\right)\right)^{2}+V\left(\vec{r}_{1}-\vec{r}_{2}\right),
$$

$V\left(\vec{r}_{1}-\vec{r}_{2}\right)$ being an effective potential (equations (21) and (22)). In a magnetic field $\mathbf{B}$ applied along the $z$-direction (we choose $\mathbf{A}\left(\mathbf{r}_{i}\right)=\left(\mathbf{B} \times \mathbf{r}_{i}\right) / 2$ ) with $\vec{r}_{i}$ lying in $(x, y)$ plane the Hamiltonian for the relative motion (see equations (15) and (16)) becomes:

$$
H=-2 \nabla^{2}+\gamma L_{z}+\frac{1}{8} \gamma^{2} r^{2}+V(r),
$$

where $\gamma=\hbar \omega_{B} / 2 R_{B}$, with $\omega_{B}=e B / m c$, and for $V(r)$ we will use both potentials (21) and (22). The trial wave function $\psi$ of the system with variational parameters $\lambda, \delta$ we have chosen in the form :

$$
\psi=N \exp \left(-\lambda r^{2}\right)(1+\delta r) \text {. }
$$

For the ground state energy $E$ of the 2D system with potential described by equation (21), we obtain

$$
\begin{gathered}
E=8 \lambda+A^{-1}\left\{S_{1}+S_{2}\right\} ; \\
S_{1}=\int_{0}^{a_{O_{v}}}\left\{\left(\left(\gamma^{2} / 8-8 \lambda^{2}+1 / a_{O v}^{3}\right) r^{3}+2-3 r / a_{O v}\right)(1+\delta r)^{2}+\left(8 \lambda \delta r^{2}-2 \lambda\right)(1+\delta r)\right\} \exp \left[-2 \lambda r^{2}\right] d r, \\
S_{2}=\int_{a_{O v}}^{\infty}\left\{\left(\gamma^{2} / 8-8 \lambda^{2}\right) r^{3}(1+\delta r)^{2}+\left(8 \lambda \delta r^{2}-2 \lambda\right)(1+\delta r)\right\} \exp \left[-2 \lambda r^{2}\right] d r .
\end{gathered}
$$

For potential $V(r)$ defined by (22), we have for $E$ the same expression (26) with new $S_{1}$ and $S_{2}$ :

$$
\begin{gathered}
S_{1}=\int_{0}^{a_{N}}\left\{\left(\left(\gamma^{2} / 8-8 \lambda^{2}\right) r^{3}+2-\frac{4 a_{N} r}{r_{s}^{2}}{ }_{1} F_{2}\left(\frac{1}{2},-\frac{1}{2} ; 1 ; \frac{r^{2}}{a_{N}^{2}}\right)\right)(1+\delta r)^{2}+\left(8 \lambda \delta r^{2}-2 \lambda\right)(1+\delta r)\right\} \exp \left[-2 \lambda r^{2}\right] d r \\
S_{2}=\int_{a_{N}}^{\infty}\left\{\left(\gamma^{2} / 8-8 \lambda^{2}\right) r^{3}(1+\delta r)^{2}+\left(8 \lambda \delta r^{2}-2 \lambda\right)(1+\delta r)\right\} \exp \left[-2 \lambda r^{2}\right] d r .
\end{gathered}
$$

\section{§3. Discussion}

We have chosen the ground state trial wave function as a product of a Gaussian function and a simple polynomial function $\exp \left(-\lambda r^{2}\right)(1+\delta r)$, to describe Coulomb repulsive interaction of two particles in parabolic potential, and a Bessel function to take into account the potential barrier on the QD surface. The factor $g(r, \theta)=\exp \left(-\gamma r^{2} \sin ^{2} \theta / 4\right)$ (see equation (13)) describes the influence of the magnetic field. A function similar to $\exp \left(-\lambda r^{2}\right)(1+\delta r)$ was obtained by Taut $[6,19]$ to describe 
the behaviour of two electrons interacting with Coulomb potential in an external infinite range harmonic oscillator potential (hereafter we will write harmonic potential). Our variational function in the absence of magnetic field and for large values of the QD radius $(d \rightarrow \infty)$ leads to the same function obtained in [6]. On the other hand, in the presence of magnetic field (in the absence of Coulomb interaction) the ground state energy calculated on the basis of our variational approach tends for $d \rightarrow \infty$ to the exact solution [37] with the accuracy discussed in Appendix A.

The total ground state energy $E$ of the acceptor-electron system was calculated as a function of the QD radius $d$ on the basis of equations (4)-(5) in the case of the infinite potential barrier and on the basis of equations (9)-(11) in the case of finite barrier (with $U_{0}=50 R^{*}$, a typical value of the potential barrier for GaAS QDs). The minimization of $E$ with respect to variational parameters $\lambda$ and $\delta$ was performed for different values of $d$ and $\gamma_{p}$. The obtained dependences of $E$ (in units of $R^{*}$ ) on $d$ (in units of $a_{B}^{*}$ ) are shown in figure 1 (curve 1 for infinite, and curve 2, 3 for finite barrier) for fixed values of $\gamma_{p}$. The corresponding results for the ground state energy $E$ of the system in the presence of magnetic field are obtained and presented in figure 1 (curves 4, 5).

The correlation energy (the energy of Coulomb interaction of two equally charged particles) is defined by the expression $E_{c}=E-E_{f}$, where $E_{f}$ is the confinement energy of the system (the energy in the absence of repulsive Coulomb interaction). For calculation of the energy $E_{f}$ we used the same equations (2), (7), and (13) for wave functions but with variational parameter $\delta=0$. In figure 2 we present the dependences of correlation energy $E_{c}$ on the radius $d$ in the cases of infinite (curve1) and finite (curves 2-5) potential barriers for different values of magnetic field.

[Insert figure 1 about here]

We have obtained a strong increase of $E_{c}$ with the decrease of $d$ in the case of infinite barrier (figure 2, curve 1). As seen in figure 2, the correlation energy (curve 2) for the finite barrier increases at a slower rate in comparison with the case of infinite potential.

[Insert figure 2 about here]

When $d$ becomes very large the difference between the total energies of the system for the two cases of finite and infinite barriers tends to zero with the energy tending to the corresponding value for the system with harmonic potential [6]. For the small values of the QD radius $(d \approx 1)$, in the range of $\gamma_{p} \leq 3$, we approach to the case of square well with $U_{0}>>\gamma_{p}^{2} d^{2}$ : since $E>\gamma_{p}^{2} d^{2}$, the particle is localized mainly by the "square " finite or infinite well and $E_{c}$ is sensitive to the model of the potential barrier. On the contrary, the increase of the correlation energy with decrease of $d$ is very slow for larger values of $\gamma_{p}\left(\gamma_{p}>3\right)$ (see figure 2, curves 3-5). We have found also that for $\gamma_{p}>3$ the correlation energy is less sensitive to the model of potential barrier even for $d \approx 1$ (see table 1 ). The particle now is localized mainly by the parabolic potential and it does not feel the existence of the infinite or finite barrier on the QD surface.

[Insert table 1 about here]

The presence of the magnetic field leads to the additional increase of the correlation energy of the system (see figure2).

We now focus on a cut-parabolic (without the step profile in the QD surface) potential of the form $U_{0}=\gamma_{p}^{2} d^{2}$. The dependence of the total energy $E$ of the acceptor-electron system on $\gamma_{p}$ in this case is shown in figure 3 for $d=3$ and different values of the magnetic field.

[Insert figure 3 about here]

The dependence of $E_{c}$ for the acceptor-electron system on $\gamma_{p}$ in QD with finite potential barrier $U_{0}=\gamma_{p}^{2} d^{2}$ is presented in figure 4 for $d=3$ and different values of magnetic field.

[Insert figure 4 about here] 
It is interesting to compare the correlation energy for the cut-parabolic QD with the energy for the case of infinite depth harmonic potential obtained on the basis of equation (13) with $d \rightarrow \infty$. In table 2 we present the energies obtained for different parameters of the problem in the cases when the total energy of the system is about $80 \%$ of the finite barrier. For these values of parameters the energy level becomes too shallow and the QD is approaching ionization.

[Insert table 2 about here]

We find a small difference between $E_{c}$ for the cases of harmonic and cut-parabolic potential. For example, for $d=3, \gamma_{p}=0.7, \gamma=0$ the relative difference $\alpha$ for $E_{c}$ is about $3 \%$. This can be qualitatively explained by the fact that the Coulomb interaction energy behaves as $E_{c} \sim 1 / \bar{r}$ (with $\bar{r}$ the expectation value of coordinate $r$ ). Because of the vicinity of corresponding values of the confinement energy $E_{f}$ for harmonic and $E_{f}^{\prime}$ for cut-parabolic potential (see Appendix B) the squeezing of the particle towards the acceptor is approximately the same in these two cases, with $\bar{r} \approx 1.6$, as it actually turns out from a straightforward calculation on the basis of equation $(7,13)$.

We notice some increase of $\alpha$ for smaller values of QD radius (see table 2): with decrease of $\bar{r}$ the correlation energy $E_{c} \sim 1 / \bar{r}$ becomes more sensitive to the choice of the potential barrier form.

It is well known that in a shallow spherical QD the presence of a magnetic field gives rise to a bound state (see Bychkov's problem in [32]). Now, when the bound state already exists in QD, we obtain a considerable decreasing of $\alpha$ due to the additional magnetic field confinement (see figure 4, curve 3); for $\gamma=3, \gamma_{p}=1 d=3 \alpha$ is about $2 \%$.

On the contrary, in the case approaching the square well, when $U_{0}>\gamma_{p}^{2} d^{2}$ the relative difference for $E_{c}$ increases. For example, for $\gamma_{p}=3, d=1.1, \alpha$ is about $10 \%$, although the level is deeper in this well model: $E / U_{0} \approx 0.3$ (see table 1). This is because the confinement energies for the infinite square well $E_{f}=11.09 R^{*}$ and finite one $E_{f}{ }^{\prime}=9.95 R^{*}$ are quite different. The particle is squeezed better by the infinite barrier and this corresponds to increase of the correlation energy.

In the absence of Coulomb interaction and magnetic field, the problem can be solved exactly (Appendix B). To estimate the accuracy of variational method, we compare our results in the absence of Coulomb interaction with the exact solution. The maximal difference between the energies is expected near the critical values of QD parameters: for $d=3, \gamma_{p}=0.4$ (when $E / U_{0} \approx 0.8$ ) we have found that the relative difference between the results obtained on the basis of our variational approach and the exact solution is $3 \%$ (Appendix B). Because of the similarity of the trial wave function forms for the system without and with Coulomb interaction, we conclude that the upper bound of the error is also about 3\% when the Coulomb repulsive interaction is taken into account. Obviously, the variational method has better accuracy when the system is far from the critical point.

We found the total ground state energy $E$ of the relative motion of the two-electron system in QD with harmonic potential in the presence of magnetic field using equation (19). The correlation energy is still defined by the expression $E_{c}=E-E_{f}$, where the second term is the energy of the system in the absence of Coulomb interaction between the two electrons. The obtained curves for $E_{c}$ as a function of $\gamma_{p}$ are shown in figure 5 for different values of magnetic field (in units $R^{*}=e^{2} / 2 \kappa a_{B}^{*}$ and $a_{B}^{*}=\hbar^{2} \kappa / m^{*} e^{2}$ used for acceptor-electron system). Using our variational functions (see equation (18)) we obtained that the value of $E_{c}$ is less than the corresponding value of correlation energy for the acceptor-electron system with harmonic potential (see table 3 ).

[Insert table 3 about here] 
Since the correlation energy in the two-electron system is lower, the expectation value for the relative distance $\bar{r}$ between the two electrons is larger in comparison with the case of the acceptorelectron system. We find, therefore, that in the two-electron system the sensitivity of the correlation energy $E_{c}\left(E_{c} \sim 1 / \bar{r}\right)$ to potential barrier model (harmonic or cut-parabolic) must be lower than in the case of acceptor-electron system. For example, in the two-electron system, $\alpha$ will be less than $3 \%$ in QD with parameters $\gamma_{p}=0.7, \gamma=0$ and $d=3$ (see table 2). For this system, in the presence of the magnetic field with $\gamma=3, \alpha$ is expected to be less than $2 \%$ in QD with $d=3$ and $\gamma_{p}=1$.

These results can be explained by the fact that the Coulomb correlation energy $E_{c}$ has a maximum when one particle is fixed at the center of the QD [38]. In other case (when a charged particle is not fixed at the centre) $E_{c}$, as well as the difference between the energies for various potentials are both expected to decrease. The obtained dependences $E_{c}(\gamma)$ are shown in figure 6 for different values of $\gamma_{p}$. The increase of $E_{c}(\gamma)$ is slower in comparison with the $E_{c}\left(\gamma_{p}\right)$ dependence (figure 5), since the main confinement is due to the QD parabolic potential. The increase of $E_{c}(\gamma)$ for $\gamma_{p}=3$ is much slower than for $\gamma_{p}=1$ (see figure 6). This is because for $\gamma_{p} \geq 3$ we have strong parabolic confinement, and in this region the energy is not so sensitive to the magnetic field.

[Insert figure 5 about here]

[Insert figure 6 about here]

Finally, the total ground state energy $E$ of the relative motion of two electrons in the screened 2D Coulomb system in the presence of an external perpendicular magnetic field was found from equations (26), (27) for the Overhauser model with a neutral choice of disc, and on the basis of equations (26), (28) with a choice based on Nagy's procedure.

The obtained curves for energy $E(\gamma)$ are shown in figure 7 for different values of $r_{s}$, and for the correlation energy $E_{c}(\gamma)$ in figure 8. For comparison, curves for the case of bare Coulomb interaction between the two electrons (curve 5) in both figure 7 and figure 8 are also shown. We see that correlation energy is larger for the case of the bare Coulomb interaction in comparison with the case of the screened interaction. The bare Coulomb potential actually offers an upper bound, corresponding to the low-density limit $r_{s} \rightarrow \infty$.

In the case of Coulomb interaction in QDs, inclusion of the variational parameter $\delta$ led to the lowering of energy by $2 \%$. Now, in the case of uniform electron gas we have real electrons in vacuum with the bare electron mass. The electrons are confined only by magnetic field, and the magnetic length (for real electron) cannot be much smaller than the Bohr radius $(\gamma \sim 1)$ even for strong magnetic fields (experimentally accessible). Therefore, even for the strongest localization by a magnetic field, the wave function is more extended in the screened electron gas model in comparison with the QD case. The anharmonicity due to Coulomb interaction now is the strong factor; we obtain that inclusion of $\delta$ results in energy lowering by about $6 \%$.

[Insert figure 7 about here]

[Insert figure 8 about here]

\section{§4. Conclusion}

In this work a variational method was developed for the investigation of Coulomb correlation between two equally charged particles in systems with radial confinement in an external homogeneous magnetic field. The proposed variational wave functions take into account the Coulomb repulsive interaction, as well as the potential barrier on the QD surface.

The focus in particular was on spherical parabolic QDs with various boundary conditions, as well as on circular magnetically confined states in the Overhauser screening model of a many electron system. We found that the correlation energy is relatively insensitive to the choice of infinite range 
harmonic or the cut-parabolic potential of the QD even for energies quite close to the ceiling of the potential barrier. On the contrary, when confinement is mainly due to an infinite or finite square well the energy is quite sensitive to the choice of different confinement conditions. The type of variational wave functions used involved an anharmonicity parameter (beyond Gaussian behaviour) and also appropriate use of Bessel functions, according to the case. For the confined Overhauser screening model we obtained a strong decrease of correlation energy in comparison with the bare Coulomb potential, as well as a dominant role of anharmonicity in energy lowering compared to the QD cases. The results obtained, can be useful when examining the optical properties of real QDs with different potential barriers in device and biomedical applications [39].

Acknowledgements A.P.Djotyan acknowledges support by Armenian State Programme 'Semiconductor Nanoelectronics'.

\section{Appendix A}

The Hamiltonian of an electron in the field of harmonic potential in the presence of a homogeneous magnetic field $\mathbf{B}$ applied along the $z$-direction (we choose $\mathbf{A}(\mathbf{r})=(\mathbf{B} \times \mathbf{r}) / 2)$ ) in cylindrical coordinates becomes

$$
H=-\hbar^{2} \nabla^{2} / 2 m^{*}+\frac{i e \hbar B}{2 m^{*} c} \frac{\partial}{\partial \varphi}+\frac{1}{2} m^{*} \omega_{p}^{2} z^{2}+\left(m^{*} \omega_{p}^{2} / 2+\frac{e^{2} B^{2}}{8 m^{*} c^{2}}\right) \rho^{2} .
$$

The variables for this case are decoupled, and the problem is exactly soluble [37] with the energy:

$$
\varepsilon_{n_{1} m n_{2}}=-\frac{e \hbar B m}{2 m^{*} c}+\hbar\left(2 n_{1}+|m|+1\right) \sqrt{\omega_{p}^{2}+\frac{e^{2} B^{2}}{4 m^{* 2} c^{2}}}+\hbar \omega_{p}\left(n_{2}+1 / 2\right), n_{1}, n_{2}=0,1, . ; m=0, \pm 1, . .
$$

For $\left(\gamma=3, \gamma_{p}=1\right)$ the ground state energy $\left(n_{1}=n_{2}=m=0\right)$ obtained on the basis of equation (A2) is $E_{f}=4.61 R^{*}$. For the same values of parameters we have obtained $E_{f}=4.74 R^{*}$ on the basis of the variational method (see equation (13) with $\delta=0, d \rightarrow \infty$ ); the relative difference between this result and the exact solution is $2.8 \%$. For stronger parabolic confinement and weaker magnetic one, the energy of the system calculated on the basis of our variational functions is closer to the exact solution. For example, for $\gamma=1, \gamma_{p}=2$ the ground state energy obtained on the basis of equation (A2) is $E_{f}=6.12 R^{*}$ and $E_{f}=6.16 R^{*}$ on the basis of the variational method; now the relative difference is $0.6 \%$.

\section{Appendix B}

The ground state wave function and the energy of an electron in a spherical QD under a parabolic potential and confined with a finite potential $U_{0}=\gamma_{p}^{2} d^{2}$ (see equation (6)) on the surface of QD, for $r<d$ have the form [40]:

$$
f_{1}(r)=C_{1} \exp \left(-\gamma_{p} r^{2} / 2\right)_{1} F_{1}\left(-\alpha_{1}, 3 / 2, \gamma_{p} r^{2}\right), E_{f}=\gamma_{p}\left(4 \alpha_{1}+3\right)
$$

where ${ }_{1} F_{1}$ is the confluent hypergeometric function. For $r \geq d$ we have

$$
f_{2}(r)=C_{2} K_{1 / 2}\left(\beta_{1} r\right) / r^{1 / 2}, \beta_{1}^{2}=U_{0}-E_{f} .
$$

The parameter $\alpha_{1}$ and the energy $E_{f}$ of the system can be obtained from the condition of equality of logarithmic derivatives at $\mathrm{r}=\mathrm{d}$. For $d=3, \gamma_{p}=0.4\left(U_{0} \approx 1.44 R^{*}\right)$ the energy obtained on the basis of equations (B1), (B2) is $E_{f}=1.14 R^{*}$ with $E_{f} / U_{0} \approx 0.8$. For the same values of parameters we have obtained $E_{f}=1.18 R^{*}$ on the basis of the variational method (from equation (7) with $\delta=0$ ). Thus, near the QD ionization the relative difference between the results obtained on the basis of the variational approach and the exact solution is 3\%. For larger values of $\gamma_{p}$ the energy for the harmonic case is very close to the energy of the cut-parabolic one. For example, for $d=3$, 
$\gamma_{p}=0.7\left(U_{0} \approx 4.41 R^{*}\right)$ we obtain $E_{f}=2.09 R^{*}$ from equations (B1), (B2) with $E_{f} / U_{0} \approx 0.5$. In the case of harmonic oscillator potential we obtain the ground state energy from equation (B1) with $\alpha_{1}=0$; for $\gamma_{p}=0.7$ we have $E_{f}=3 \gamma_{p}=2.1 R^{*}$.

\section{References}

1. Shabaev, A., Efros, Al.L., Gammon, D., and Merkulov, I.A., 2003, Phys. Rev. B, 68, 201305.

2. Tischler, J.G., Bracker, A. S., Gammon, D., and Park, D., 2002, Phys. Rev. B, 66, 081310.

3. Ciorga., M., Sachrajda, A.S., Hawrylak, P., Gould, C., Zawadzki, P., Jullian, S., Feng, Y., and Wasilewski, Z., 2000, Phys. Rev. B, 61, R16315.

4. Riva, C., Escorcia, R.A., Govorov, A.O., and Peeters, F.M., 2004, Phys. Rev. B, 69, 245306.

5. Hartmann, A., Ducommun, Y., Kapon, E., Hohenester, U., and Molinari, E., P., 2000, Phys. Rev. Lett., 84, 5648.

6. Taut, M., 1993, Phys. Rev. A, 48, 3561.

7. Taut, M., Ernst, A., and Eschrig, H., 1998, J. Phys. B, 31, 2689.

8. Fominykh, N., Kudin, O., Ernst, A., and Berakdar J., 2003, J.Phys.B, 36, 1.

9. Pfannkuche, D., and Gerhardts, R.R., 1991, Phys. Rev. B, 44, 13132.

10. Pfannkuche, D., and Gerhardts, R.R., 1993, Physica B, 7, 189.

11. Maksym, P.A., Imamura, H., Mallon,G.P. and Aoki, H., 2000, J. Phys: Cond.Matt.,12, R299

12. Kouwenhoven, L.P., Austing, D.G, and Tarucha, S., 2001, Rep. Prog. Phys., 64, 701.

13. Kouwenhoven, L.P., Oosterkamp, T.H., Danoesastro, M.W.S., Eto, M., Austing, D.G., Honda, T., and Tarucha, S., 1997, Science, 278, 1788.

14. Matulis, A., and Peeters, F.M., 2001, Solid State Commun., 117, 655.

15. Reimann, S.M., Koskinen, M., and Manninen, M., 2000, Phys. Rev. B, 62, 8108.

16 Maksym, P.A. and Chakraborty, T., 1990, Phys. Rev. Lett., 65, 108.

17 Merkt, U., Huser, J., and Wagner, M., 1991, Phys. Rev. B, 43, 7320.

18 Wagner,M., Merkt, U., and Chaplik, A.V.,1992, Phys. Rev. B, 45, 1951.

19 Taut, M., 1994, J.Phys.A, 27, 1045.

20 Dineykhan, M., and Nazmitdinov, R.G., 1997, Phys. Rev. B, 55, 13707.

21 Zhu, J. -L., Zhu, Z., Kawazoe, Y., and Yao T., 1999, J. Phys.:Condens. Matter, 11, 229.

22 Simonovic, N.S. and Nazmitdinov, R.G., 2003, Phys.Rev. B, 67, 041305.

23 Ralko, A., and Truong T.T., 2004, Physics Letters A, 323, 395.

24 Bednarek, S., Szafran, B., and Adamowski, J., 1999, Phys. Rev. B, 59, 13036.

25 Peeters, F.M., and Schweigert, V.A., 1996, Phys. Rev. B, 53, 1468.

26 Overhauser, A.W., 1995, Can. J. Phys. 73, 683.

27 Gori-Giorgi, P., and Perdew J.P., 2001, Phys. Rev. B, 64, 155102.

28 Moulopoulos, K., and Ashcroft, N.W., 1993, Phys. Rev. B, 48, 11646.

29 Polini, M., Moulopoulos, K., Davoudi, B., and Tosi, M.P., 2002, Phys. Rev. B, 65, 165306.

30 Tavernier, M.B., Anisimovas E., Peeters, F.M., Szafran, B., Adamowski, J., and Bednarek, S., 2003, Phys. Rev. B, 68, 205305.

31 Avetisyan, A.A., Djotyan, A.P., and Kazaryan, E.M., 2003, Phys. Stat. Sol (c), 0, 734.

32 Landau, L.D., and Lifshits, E.M., 1981, Quantum Mechanics (Pergamon Press). p. 459.

33 Xiao, Z., Zhu, J., and He, F., 1996, J. Appl. Phys., 79, 9181.

34 Moreno, J., and Marinescu, D.C., 2003, J. Phys.: Condens. Matter, 15, 6321.

35 Polini, M., Sica, G., Davoudi, B., and Tosi, M.P., 2001, J. Phys.: Condens. Matter, 13, 3591.

36 Nagy, I., 1999, Phys.Rev. B, 60, 4404.

37 Galitskiy, V.M., Karnakov, B.M., and Kogan, V.I, 1981, Zadachi po kvantovoy mekhanike ( Moskcow, Nauka). p.51.

38 Brown, J.W. and Spector H.N., 1986, J. Appl. Phys., 59, 1179.

39 Lalayan, A., Avetisyan, A., and Djotyan, A., 2005, Laser Phys. Lett., 2, 12.

40 Xiao, Z., Zhu, J., and He, F. J., 1996, Superlattices and Microstructures, 19, 137. 
Table 1. The relative difference $\left(E_{c \infty}-E_{c}\right) / E_{c \infty}$ for correlation energy in $Q D(d=1.1)$, ( $E_{c \infty}$ corresponds to infinite barrier case).

\begin{tabular}{|l|c|c|c|c|c|}
\hline$U\left(R^{*}\right)$ & $\gamma_{p}$ & $E\left(R^{*}\right)$ & $E_{c}\left(R^{*}\right)$ & $E_{f}\left(R^{*}\right)$ & $\left(E_{c \infty}-E_{c}\right) / E_{c \infty}$ \\
\hline$\infty$ & 3 & 15.67 & 4.58 & 11.09 & \multirow{2}{*}{$10 \%$} \\
\hline 50 & 3 & 14.07 & 4.12 & 9.95 & \multirow{2}{*}{$7.6 \%$} \\
\hline$\infty$ & 4 & 17.97 & 4.79 & 13.18 & \\
\hline 50 & 4 & 16.83 & 4.42 & 12.41 & \\
\hline
\end{tabular}

Table 2. The relative difference $\alpha=\left(E_{c \infty}-E_{c}\right) / E_{c \infty}$ in cut-parabolic QD near ionization $\left(E / U_{0} \approx 0.8\right) ; E_{c \infty}$ corresponds to harmonic potential.

\begin{tabular}{|c|c|c|c|c|c|}
\hline$d$ & $\gamma_{p}$ & $U_{0}=\gamma_{p}^{2} d^{2}$ & $\gamma$ & $E_{c}\left(R^{*}\right)$ & $\left(E_{c \infty}-E_{c}\right) / E_{c \infty}$ \\
\hline 3 & 0.7 & 4.41 & 0 & 1.66 & \multirow[b]{2}{*}{$3 \%$} \\
\hline$d \rightarrow \infty$ & 0.7 & $\infty$ & 0 & 1.71 & \\
\hline 1 & 5 & 25 & 0 & 4.68 & \multirow[b]{2}{*}{$3.5 \%$} \\
\hline$d \rightarrow \infty$ & 5 & $\infty$ & 0 & 4.85 & \\
\hline 3 & 1 & 9 & 3 & 2.51 & \multirow[b]{2}{*}{$2 \%$} \\
\hline$d \rightarrow \infty$ & 1 & $\infty$ & 3 & 2.56 & \\
\hline
\end{tabular}

Table 3. The correlation energies $E_{c}^{\prime}$ (for electron-electron) and $E_{c}$ ( for acceptor-electron) interaction $(d \rightarrow \infty)$

\begin{tabular}{|l|l|l|l|}
\hline$\gamma_{p}$ & $\gamma$ & $E_{c}^{\prime}$ & $E_{c}$ \\
\hline 3 & 3 & $2.75 R^{*}$ & $3.92 R^{*}$ \\
\hline 0.7 & 0 & $1.2 R^{*}$ & $1.71 R^{*}$ \\
\hline 5 & 0 & $3.43 R^{*}$ & $4.85 R^{*}$ \\
\hline
\end{tabular}




\section{Figure captions}

Figure 1. The total energy $E$ of the acceptor-electron system (in units of $R^{*}$ ) as a function of $d$ (in units $a_{B}^{*}$ ) for different values of magnetic field: a) infinite barrier:1) $\gamma_{p}=3, \gamma=0$ and b) finite potential barrier $U_{0}=50 R^{*}$ :

2) $\gamma_{p}=3, \gamma=0$;3) $\gamma_{p}=4, \gamma=0$;4) $\gamma_{p}=4, \gamma=2$; 5) $\gamma_{p}=4, \gamma=4$.

Figure 2. The correlation energy $E_{c}$ of the acceptor-electron system (in units of $R^{*}$ ) as a function of $d$ (in units $a_{B}^{*}$ ) for different values of magnetic field: a) infinite barrier: 1) $\gamma_{p}=3, \gamma=0$ and b) finite potential barrier $U_{0}=50 R^{*}$ : 2) $\gamma_{p}=3, \gamma=0$;3) $\gamma_{p}=4, \gamma=0$;4) $\gamma_{p}=4, \gamma=2$; 5) $\gamma_{p}=4, \gamma=4$.

Figure 3. The total energy of the acceptor-electron system (in units of $R^{*}$ ) in QD $(d=3)$ with finite potential barrier $U_{0}=\gamma_{p}^{2} d^{2}$ as a function of $\gamma_{p}$ for different values of magnetic field: 1) $\gamma=0$;2) $\gamma=1$;3) $\gamma=3$.

Figure 4. The correlation energy of the acceptor-electron system (in units of $R^{*}$ ) in QD $(d=3)$ with finite potential barrier $U_{0}=\gamma_{p}^{2} d^{2}$ as a function of $\gamma_{p}$ for different values of magnetic field: 1) $\gamma=0$;2) $\gamma=1$;3) $\gamma=3$.

Figure 5. The correlation energy of two-electron system (in units of $R^{*}$ ) in parabolic QD as a function of $\gamma_{p}$ for different values of magnetic field: 1) $\gamma=1$; 2) $\gamma=3$.

Figure 6. The correlation energy of two-electron system (in units of $R^{*}$ ) in parabolic QD as a function of $\gamma$ for different values of parabolic potential: 1) $\gamma_{p}=1$;2) $\gamma_{p}=3$.

Figure 7. The total ground state energy $E$ of 2D screened system as a function of $\gamma$ for different values of $r_{s}$ (in units of $R_{B}$ ). The dotted curves are for Nagy's potential with $r_{s}=1$ (lower curve) and $r_{s}=2$ (upper curve). The dashed curves are for Overhauser potential with $r_{s}=1$ (lower curve) and for $r_{s}=2$ (upper curve). The solid curve is for bare Coulomb interaction.

Figure 8. The correlation energy of 2D screened system (in units of $R_{B}$ ) as a function of $\gamma$ for different values of $r_{s}$ 1) $r_{s}=1$, Nagy's potential; 2) $r_{s}=1$, Overhauser potential; 3) $r_{s}=2$, Nagy's potential; 4) $r_{s}=2$, Overhauser potential; 5) bare Coulomb interaction. 
Figures

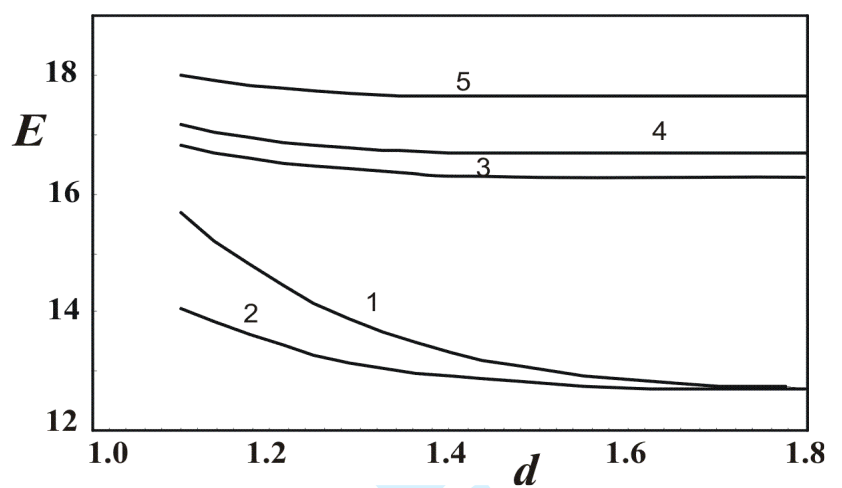

Figure 1.

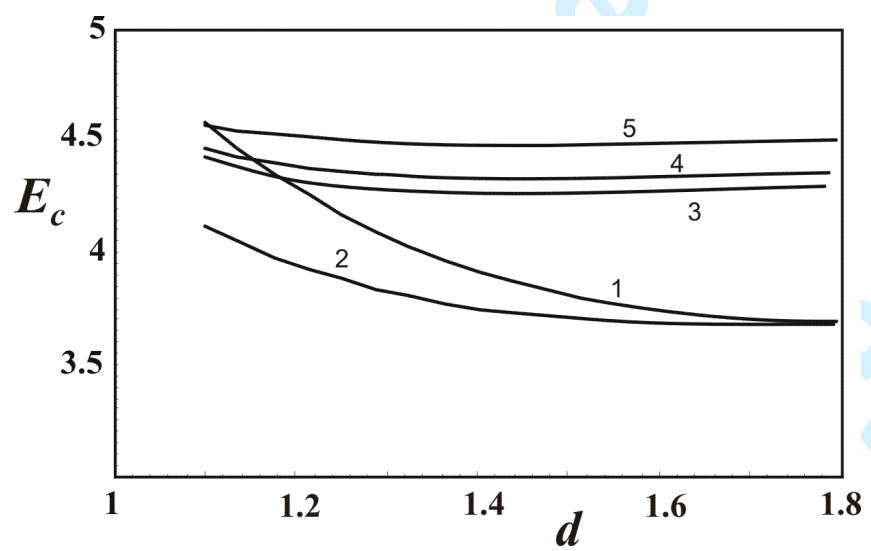

Figure 2.

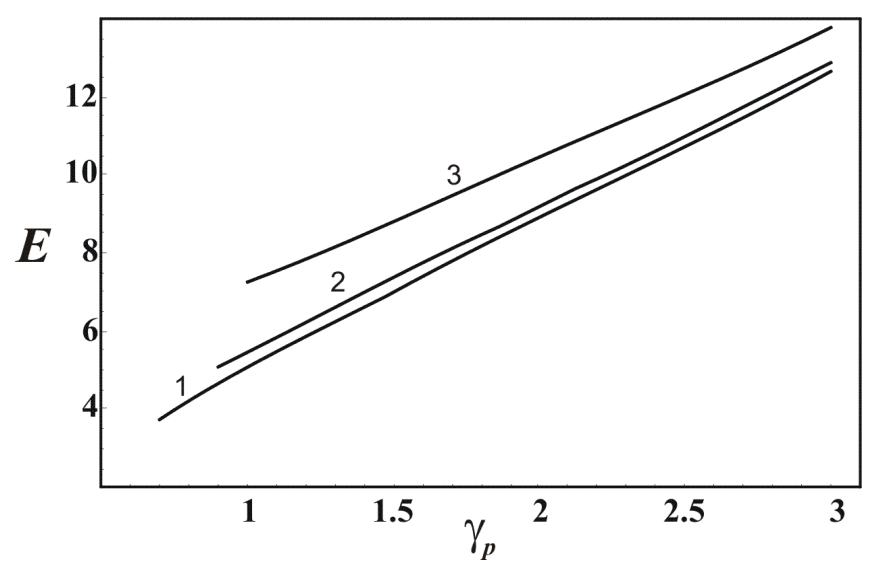

Figure 3. 


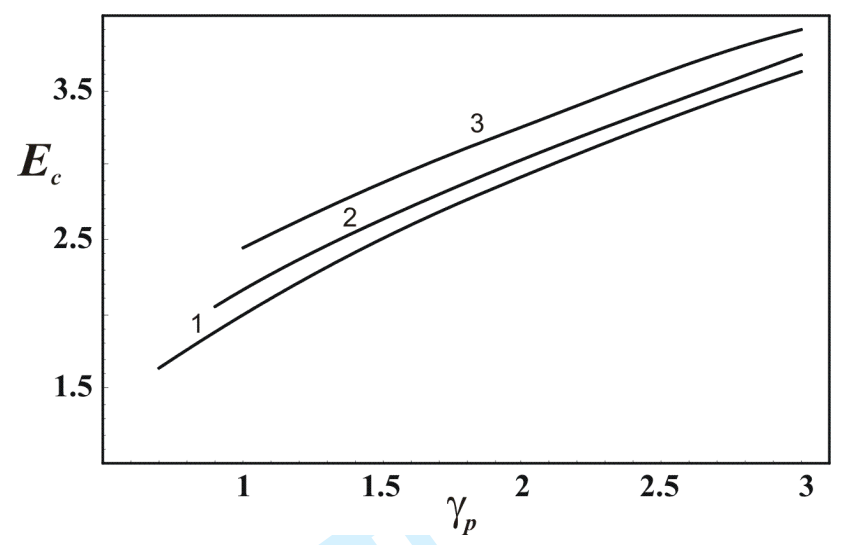

Figure 4.

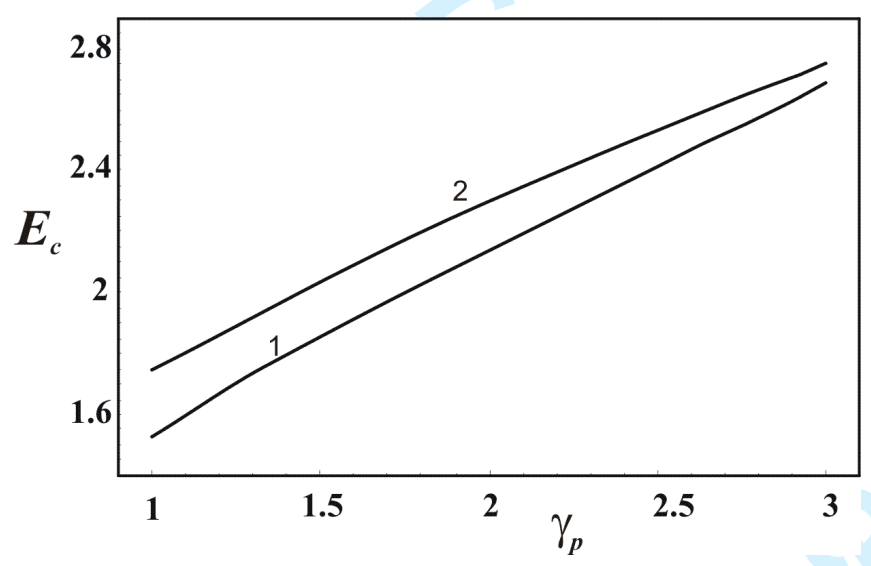

Figure 5.

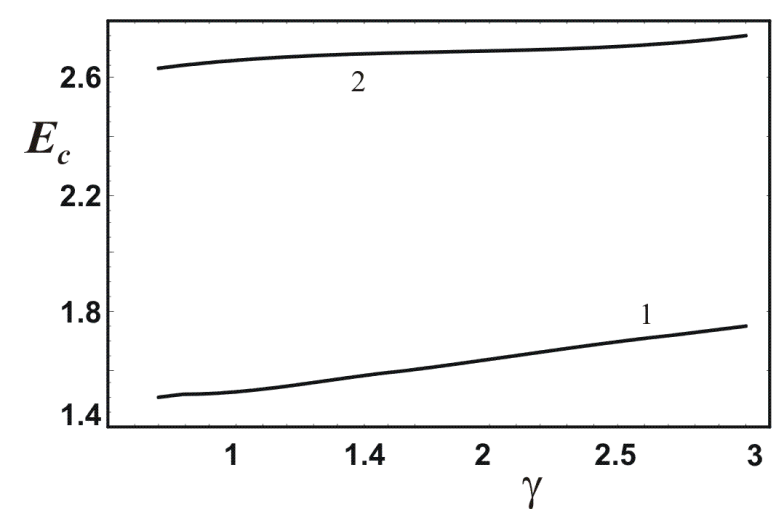

Figure 6. 


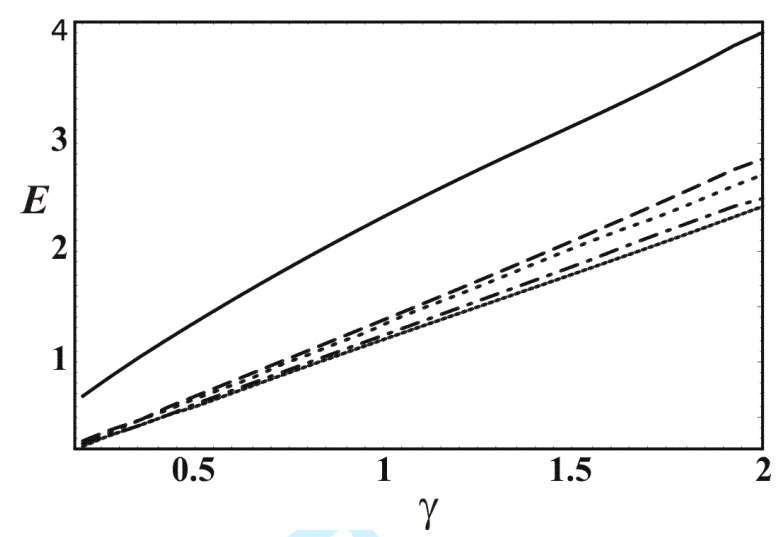

Figure 7.

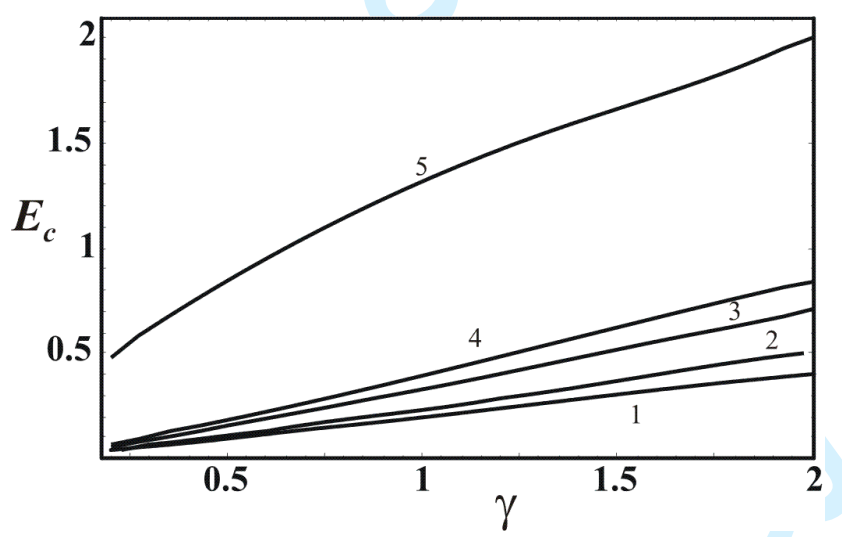

Figure 8. 